\title{
Testing the BOSS hypothesis: Evidence for position-insensitive orthographic priming in the lexical decision task
}

\author{
TIMOTHY RICHARD JORDAN \\ University of Reading, Reading, England
}

\begin{abstract}
One recent conceptualization of the process of lexical access, the basic orthographic syllabic structure (BOSS) hypothesis, has been developed from a number of separate empirical and theoretical sources, and implicates distinct characteristics of the word recognition process. Using a lexical decision/priming paradigm, the present study tested all such characteristics simultaneously, together with a control condition in which simple sequences of letters were repeated within pairs of words, occupying different serial positions in each member of a pair. No evidence was obtained to suggest that BOSSs enjoy a special psychological status. Yet evidence from the same experiment suggests that words are processed via multiletter units in the lexical decision task, and that these units are not position specific, because they produce facilitation even when presented in different serial positions across primes and targets. Two interpretations of this position-insensitive orthographic priming are presented.
\end{abstract}

To recognize and understand a written word, the reader must encode physical characteristics of that word and match them with an appropriate representation in his/her long-term word store, or internal lexicon. The precise nature of this encoding process has been a predominant issue in psycholinguistic research for the past decade or so, and, in that time, several types of encoding have been suggested.

According to some researchers (e.g., Meyer, Schvaneveldt, \& Ruddy, 1974; H. Rubenstein, Lewis, \& M. A. Rubenstein, 1971; H. Rubenstein, Richter, \& Kay, 1975), the recognition of a word depends upon the conversion of its orthographic content into a phonological code, because it is believed that it is this code that enables lexical access to occur. According to others (e.g., Gibson \& Guinet, 1971; Murrell \& Morton, 1974; Taft \& Forster, 1975), the access code is of a morphological form: when the reader is presented with a polymorphemic word (e.g., returned), the code derived is the root morpheme (turn), and stored permissible prefixes and inflections are accessible via this lexical representation. Finally, according to researchers such as Hansen and Rodgers (1968) and Spoehr and Smith (1973), the encoding of a word involves its syllabification into appropriate syllabic structures, structures which then form the necessary code

This work was supported by a Bitish Science and Engineering Research Council postgraduate studentship and by Grant No. G8408210N from the British Medical Research Council. Many thanks to Kenneth Forster, Leslie Henderson, and two anonymous reviewers for their help in fine-tuning my initial efforts. Part of this work was carried out while the author was visiting the University of Calgary, Calgary, Alberta, Canada

Requests for reprints should be sent to Timothy R. Jordan, Department of Psychology, University of Reading, Whiteknights, Reading, RG6 $2 \mathrm{AL}$, England. through which access of an appropriate lexical representation is achieved.

More recently, in 1979, Taft introduced an interesting view of the lexical access procedure; he posited an access code, derived purely from the orthography of a stimulus, to which he gave the title basic orthographic syllabic structure (BOSS; Taft, 1979a). Like many other hypotheses, the BOSS hypothesis claims that lexical access involves a visual (rather than phonological) encoding process; it is in its explanation of the more intimate aspects of this process that it differs from other hypotheses. Taft proposed, primarily, that the printed word is encoded via a process of orthographic syllabification; that is, whole words are represented in the lexicon as syllables constructed not within articulatory or purely morphemic constraints (although morphological consideration is an intrinsic component of the BOSS hypothesis), but within the constraints of English orthography.

To present this view accurately, Taft developed a working rule whereby the appropriate syllabification for any printed word can be calculated; the rule represents a precise description of a process proposed to be automatic in normal reading: "Include in the first syllable as many consonants following the first vowel of the word as orthotactic factors will allow without disrupting the morphological structure of that word" (Taft, 1979a, p. 24). As an example of this rule, the BOSS of lantern is lant; it is not lan, because, in compliance with the BOSS hypothesis, more consonants can be added without a violation of English orthography in the resultant syllable. Furthermore, the BOSS of walrus is wal, because walr contains a terminal cluster that violates English orthography: "[WALRUS] must be syllabified as WAL RUS since the 
letter combination $L R$ is an orthographic combination that occurs neither initially nor finally in English (i.e., it violates what I will term orthotactic rules)"' (Taft, 1979a, p. 23).

Taft proposed that it is only the first syllable of a word, encountered by the proposed left-to-right, letter-by-letter analysis of the letter string, that enters into the lexical access procedure and, further, that the lexical representation of the word is simply a representation of this structuring, with information about the remaining orthographic content of the word being stored within this lexical entry. Taft concluded:

The claim that CANDLE is represented as CAND in the lexicon does not mean to imply that the full spelling of CANDLE is not stored at all. What it means is that all information about CANDLE (orthographic, phonological, semantic) is stored but that this information is accessed through an orthographic code, namely CAND, when the word is presented visually. (Taft, 1979a, p. 36)

Consequently, a single orthographic unit that in isolation is typically a nonword (e.g., CAND) is proposed to enjoy a lexical process normally resulting in the recognition of the word from which it was parsed.

Converging evidence for this proposal was provided by Taft and Forster's (1976) presentation of the BOSSs of real words (e.g., samp, the BOSS of sample; however, at the time of this earlier study, the term BOSS had not yet been developed) individually in a lexical decision experiment. Taft and Forster found that it took subjects longer to classify BOSSs as nonwords than to so classify matched nonwords that were not BOSSs of real words (e.g., selp). Taft (1979a) also capitalized on the fact that a BOSS is typically a nonword when presented in isolation; he showed that items such as ston (the BOSS of stone) incurred longer response latencies than did items such as slon, which is not the BOSS of any real word.

This, then, is the orthographic aspect of the BOSS hypothesis; but the detection of this type of syllable is critically dependent upon the morphological structure (or, more precisely, the apparent morphologial structure) of the word in which it is present. In particular, Taft proposed that the BOSS of a word is parsed only after the removal of any prefix contained in the word, and that this prefix-stripping procedure will occur for any word that starts with the necessary sequence of letters (e.g., re), even if this sequence is not a true prefix in a particular word (e.g., relish). In support of this idea, there is some evidence to suggest that the prefixes of words are removed automatically, irrespective of the appropriateness of this procedure. Taft (1979b) found that the stems of words appear to enjoy a frequency effect independent of the surface frequency of the actual words in which they appear. For example, although the words reproach and dissuade possess the same frequency of written occurrence, the frequency of the stem proach is far higher than that of suade. Taft (1979b) found that words with high-frequency stems (e.g., reproach) were recognized more quickly than were words matched for surface frequency yet with lower stem frequencies (e.g., dissuade). This suggests both that such items are stored in their decomposed form and that recognition of the words in which they are present is accomplished by the removal of prefixes and the entering of the remaining stem into the lexical access procedure. Furthermore, both Rubin, Becker, and Freeman (1979) and Taft (1981) demonstrated that pseudoprefixed words (e.g., relish) take longer to categorize as words, in a lexical decision task, than do truly prefixed words (e.g., revive). These findings suggest that the parsing of, for example, revive into prefix and stem is conducive to the rapid access of the lexical representation for vive and its stored legal prefix re. Conversely, the recognition of pseudoprefixed words (e.g., relish) is hampered by the inappropriateness of this process for accessing the lexical representations of these monomorphemic items.

These findings appear to be robust despite (and also because of) the claim made by Rubin et al. (1979) that such a process of prefix removal is task specific, occurring in the lexical decision paradigm only when nonword filler items are also prefixed. However, Taft (1981) obtained similar effects in a naming task in which no nonword filler items were employed, making Rubin et al.'s claim implausible. Furthermore, Rubin et al. removed the effect through the removal of prefixed nonwords from their stimulus set; it is quite possible, as Taft (1981) argued, that such a stimulus selection procedure may itself induce a strategy of making a "lexical" decision merely upon the basis of the existence of a prefix. Clearly, lexical access is not required for performance in this kind of stimulus environment, in which the accurate categorization of stimuli may be achieved through the detection of their first two letters. Therefore, although lexical processes may have been responsible for the interference effects obtained by Taft (1981) in both lexical decision and naming paradigms, the disruption of these processes by strategical influences in Rubin et al.'s study may explain the absence of "normal" prefix-stripping effects in that study.

As a consequence of this evidence and its interpretations, Taft (1981) argued that, irrespective of the appropriateness of a prefix, indiscriminate prefix-stripping mechanisms in the lexicon will reveal the stem of a word, from which the BOSS is then extracted and used in the process of lexical access. Indeed, the BOSS hypothesis suggests that the inappropriateness of this procedure for a pseudoprefixed word (e.g., relish) will become manifest only after a futile attempt to access the lexical representation of its incorrectly detected BOSS (lish), resulting in the delayed latency that has been found with this type of item (Taft, 1981). When this incorrectly parsed BOSS is actually represented in the lexicon, as the real BOSS of one or more words, then rejection of the inappropriate parsing procedure for pseudoprefixed items will occur after this representation has been accessed and the store of permissible affixes has been examined (Taft, 1979a, 1981).

Finally, when several words share the same BOSS, only one psychological representation of this structure is re- 
quired for the recognition of all words in which it is present (Taft, 1985). Taft's (1979a) contention that candle will be perceived as candle only after its BOSS (cand) is detected enforces the conclusion that all words possessing that BOSS (e.g., candid, candelabra) attain lexical access via this same psychological representation (by virtue of their satisfaction of the orthographic criterion for access), but are recognized as different words because of their different orthographic representations at the level of full lexical representation.

Let us now summarize the psychological implications of the BOSS hypothesis. (1) The BOSS of any monomorphemic word is the first syllable encountered by the proposed left-to-right, letter-by-letter analysis of the stimulus. (2) When a word contains a prefix or pseudoprefix, this morphemic feature will be removed before the remainder of the word is analyzed in the same way as a nonprefixed word. (3) The same lexical representation for a specific BOSS will be employed for the detection of all stimuli within which this structure is present.

These three major components of the BOSS hypothesis have been formulated in isolation across the studies mentioned. It was the purpose of the present experiment to provide a test of their functional relationship in the recognition of a word. Because almost all of the evidence supporting the BOSS hypothesis has been accumulated in the lexical decision paradigm, this paradigm was used for the present investigation.

The specific predictions of the BOSS hypothesis lend themselves to precise empirical examination. Consider a presentation of the word demon. If the arguments presented by Taft are correct, the prefix will be detached (in this example, erroneously) from the remainder of the word, the resultant orthographic content will be analyzed, and a BOSS (mon) will be detected (Taft, 1979a, 1981, 1984). Although this procedure is inappropriate for this stimulus, its inappropriateness will become apparent only after the lexical representation for mon has been accessed and an inspection of the stored orthographic information has taken place (Taft, 1979a, 1981).

Consider now the effect of repeated access to a lexical item. Stanners, Neiser, Hernon, and Hall (1979) and Stanners, Neiser, and Painton (1979) have shown that lexical decisions to words are facilitated by prior presentation of the words' stems. Similarly, in the present investigation, if demon, for example, has been stripped of its prefix and the lexical representation for the BOSS mon has been accessed, then the threshold for mon may be temporarily lowered, thus facilitating subsequent access by the same orthographic feature, even when it appears in a different word. Consequently, if that same psychological representation is subsequently re-employed in the perception of a novel stimulus (e.g., monarch), whose BOSS is in fact that which was previously activated by demon (mon), then recognition of this novel stimulus may now be facilitated. In the lexical decision paradigm employed in the present study, response latencies to the target word monarch, for example, should be shorter when a response to demon has previously been made.
Furthermore, the activation of BOSS representations should be similar for both pseudoprefixed and truly prefixed words, provided that the resultant stem does contain a BOSS that is represented in the lexicon. For example, the truly prefixed word expire would be expected to be parsed into ex and pire. An analysis of the stem would then activate the lexical representation of the BOSS pir, through which the word expire would ultimately be recognized (assuming, of course, that the BOSS hypothesis is correct). However, pir is also the BOSS of pirate, and this word also should be recognized via this representation. Again, if the BOSS is a functional component in the process of word recognition, a response to expire should facilitate the subsequent recognition of pirate.

Consider now the stimulus lemon, which is orthographically matched to demon but is neither truly prefixed nor pseudoprefixed. The BOSS of lemon is lem, and it is this lexical representation that should be employed in the recognition of this word. As a consequence, the word monarch, presented after lemon, should enjoy little or no facilitation, because no overlap of feature extraction has occurred, inasmuch as representations of lem and mon are the two different psychological entities involved. Indeed, if no other elements of facilitation are present within $l e-$ mon, it should affect performance on monarch no more than does a matched, orthographically neutral prime ( $p e$ $d a l)$. However, if the lexical decision task involves the detection of other letter groups, less formally defined than BOSSs or morphemes, then both demon and lemon may show priming effects, and to a similar degree.

It is often assumed that the repeated use of the same perceptual processes for the detection of two verbal stimuli requires that at least some of the individual letters of these stimuli occupy identical positions in both stimuli (see Johnston \& McClelland, 1980; McClelland \& Rumelhart, 1981; Rumelhart \& McClelland, 1982). According to such theories, word recognition proceeds directly from a position-specific perception of individual letter content, and because of the gross positional differences between primes and targets in the present experiment, both demon and lemon should facilitate the recognition of monarch to no greater extent than does the prior presentation of pedal. This conceptualization of the word recognition process will be returned to later.

This, then, forms the rationale underlying the present experiment, in which three priming conditions-neutral (pedal), orthographic (lemon), and prefixed BOSS (demon)-were used to study the BOSS hypothesis. However, to reliably attribute any effect of priming to the variables under investigation, it was necessary to preclude, as far as possible, any other potential priming characteristics between primes and targets. Evidence does exist suggesting that both semantic and morphemic priming can occur between words (e.g., Henderson, Wallis, \& Knight, 1984; Meyer, Schvaneveldt, \& Ruddy, 1975; Neely, 1977; Shulman, Hornak, \& Sanders, 1978; Stanners, Neiser, Hernon, \& Hall, 1979; Stanners, Neiser, \& Painton, 1979; and perhaps Murrell \& Morton, 1974, and van der Molen \& Morton, 1979, although these two 
studies appear to have employed paradigms that invoked the more general processes of memory rather than those underlying the recognition of words). Consequently, a preliminary study was conducted in an attempt to remove these potentially confounding variables from the stimulus corpus of the main experiment. Sixteen subjects were asked to judge the semantic connections between primes and targets in two ways. The subjects were given two lists, each containing the same stimuli printed in pairs of prime and target. The prime was always the first word of a pair. Pairs were listed in a random order, which was the same on both lists for any one subject, although this order varied between subjects. Each list was formed from 31 sets of four words (one prime from each priming condition plus their target word); each set was a contender for inclusion in the stimulus corpus of the main experiment. As each prime was paired with its target, this resulted in 93 pairs of stimuli to be judged.

The subjects' tasks were slightly different for the two lists. For List 1 , the subjects were asked to judge any gross semantic connection that linked the members of a pair of words in any way. As an indication of what was required, the subjects were given the example cold, frost. For List 2, the subjects were asked to divide the first word of each pair (the prime) in any way they chose in order to achieve a "relationship of meaning" between the two words. As an example, they were given the pair resign, signal, in which the root sign shares a semantic relationship with signal. List 1 was always shown before List 2 . For both lists, the subjects were instructed to tick any word pair they regarded as related in the ways described.

Six additional pairs of words were interspersed within each list to determine whether the subjects understood the tasks and performed them correctly. In List 1, each of these additional pairs reflected a semantic relationship between the two words in their entirety (e.g., nurse, doctor). In List 2, the additional pairs reflected a semantic relationship between a part of the prime and the target (e.g., remain, mainly). The data of any subject who failed to tick five out of the six additional pairs on each list were discarded.

The data of 2 subjects who failed to meet the above criterion measure were not used in the selection of stimuli. From the judgments of the 14 remaining subjects, 24 sets of four words were selected as sharing no semantic connection or "relationship of meaning" (no subject had ticked any of the three prime-target pairings of these words in either of the two tasks). These 24 sets of words were employed as stimuli in the main experiment.

\section{METHOD}

\section{Stimuli}

The 96 word stimuli are presented in the Appendix. Each of the 24 sets of four words comprised three primes (e.g., demon, lemon, pedal) and a target word (e.g., monarch). The three priming conditions were prefixed BOSS, orthographic, and neutral. Each prime in the prefixed BOSS condition (e.g., demon) was either truly prefixed or pseudoprefixed, with a stem that formed a BOSS if processed in accord with the BOSS hypothesis (Taft, 1979a, 1981). The resultant BOSS (e.g., mon) was also the BOSS of the target word (e.g., monarch).

The orthographic feature that formed a BOSS in the prefixed BOSS condition was also present in the orthographic condition (e.g., mon in lemon), but because of the orthographic content of the remainder of the string, this feature did not now constitute a BOSS. Instead, a different BOSS (e.g., lem) should be employed for the perception of these stimuli.

No two targets shared the same BOSS.

Across prefixed BOSS and orthographic conditions, primes were matched in terms of length, consonant-vowel-consonant (CVC) structure, and, as far as possible, frequency of occurrence (although the vagaries of the English language prevented precise frequency matching). Eight of the 24 matched prefixed BOSS and orthographic pairs differed by only one letter; the remainder differed by two letters. There was only one exception to this stringent matching procedure: it proved impossible to match indent with an orthographic prime of the same CVC structure.

In each set, the neutral prime (e.g., pedal) was matched to the two other primes by word length and CVC structure. The less constraining nature of these primes also permitted a more precise matching of frequency; each neutral prime possessed a frequency of occurrence approximately equal to the mean of its two matched counterparts. For each stimulus, all frequency counts were taken from two sources (Carroll, Davies, \& Richman, 1971; Francis \& Kucera, 1982), and the mean of these two counts was regarded as the estimate of frequency for that stimulus.

For each of the 96 words in the stimulus corpus, a nonword was constructed, matched for length and CVC structure. Furthermore, the evidence provided by Rubin et al. (1979) and the criticisms made by Taft (1981) suggest that subjects may adopt a strategy of lexical decision based merely upon the presence of a prefix within a stimulus. Clearly, for the present experiment, if such a strategy were adopted by the subjects, then any potential facilitative influence present within the latter part of a prime might not become manifest. Therefore, for each of the 24 primes in the prefixed BOSS condition, each nonword possessed the same prefix as did the prime word to which it was matched. This precluded the development of a systematic strategy of lexical decision based upon the detection of a prefix. Furthermore, since all prefixed nonwords formed just one quarter of the nonword corpus, the objection raised by Rubin et al. (1979), suggesting the strategical treatment of prefixed words in a total environment of prefixed nonwords, was also avoided.

No nonword item contained a critical multiletter unit that was shared by a prime-target word pair. Thus, any priming effect observed for targets could be attributed more reliably to the multiletter unit shared by a target and its prime. Furthermore, nonword items were designed so as not to contain a BOSS or comparable multiletter unit that was repeated in another nonword item; thus, nonwords did not mimic the systematic relationship that existed between the BOSS and orthographic prime-target word pairs. This precaution was adopted to create only the minimum of repetition in the experiment as a whole.

However, because of these criteria for nonword construction, it may be argued at this point that each target in this experiment could be classified as a word merely on the basis of the presence of a multiletter unit that had been encountered by the subject earlier in the session. If such misclassifications were to occur in the present experiment, then the examination of lexical processes for which the experiment was designed might be seriously contaminated by these strategical influences. However, two aspects of the stimuli used in the experiment precluded this possibility. First, as a result of their wordlike structure, over $40 \%$ of the nonwords did share letter sequences with other nonword items, yet these shared sequences differed both in size (either two or three letters in length) 
and in position within an item. Second, and perhaps most important, because of the three priming conditions employed, $67 \%$ of the words that were shown to each subject contained critical multiletter units that had not been encountered previously in the experiment (see Design). That is to say, each of the 24 primes and the 8 targets from the neutral prime condition contained a BOSS or matched orthographic unit that was not present in a word previously shown to that subject. This prevented subjects from performing a lexical decision merely on the basis of the presence in a stimulus of a previously presented letter sequence. As a result of these two aspects of the stimulus set used, it seems implausible that subjects classified stimuli in any way other than that normally used in lexical decision experiments.

The total stimulus corpus for the experiment consisted of 96 words ( $24 \times 3$ primes and 24 targets) and 96 nonwords matched to the words in length and CVC structure. These experimental stimuli were preceded by 102 practice stimuli: 51 words and 51 nonwords constructed to match these words in length and CVC structure. Ten of these words (and hence 10 of the matched nonwords) contained prefixes.

\section{Visual Conditions}

All stimuli were presented on an off-white background, in a black, proportionally spaced lowercase font based upon the Letraset Pin Ball typeface (selected for its clarity) and adapted for computergenerated graphics. Background illumination of the CRT screen was approximately $25 \mathrm{~cd} / \mathrm{m}^{2}$; a filled matrix of dots with the same horizontal $\left(1.13^{\circ}\right)$ and vertical $\left(0.23^{\circ}\right)$ visual angles as four spaced letter $a \mathrm{~s}$ had a luminance of approximately $1 \mathrm{~cd} / \mathrm{m}^{2}$, as measured by a United Detector Technology photometer.

\section{Design}

The 72 primes were randomly distributed over three groups of stimuli, with each group containing 8 neutral primes, 8 orthographic primes, 8 prefixed BOSS primes, and all 24 target words. Within any group, no primes shared a common target, so that each of the 24 primes was specific to just one of the 24 targets. In addition to these 48 words, each group also contained the 48 nonwords matched to the word stimuli. Each group was shown to one third of the subjects, so that over all subjects each target was presented equally often in each of the three priming conditions.

For all groups, word and nonword items were intermixed in a random order that was different for each subject. The presentation of a prime preceded the presentation of its target.

To investigate the durability of any priming effect, the lag (number of intervening stimuli) between prime and target was included as an additional independent variable. For each subject, half of the primes in each of the three conditions preceded their targets by between 1 and 10 intervening trials; this was called the short lag condition. The remaining primes preceded their targets by between 11 and 94 trials ( 94 is the maximum possible lag in a list of 96 items). This was called the long lag condition. The precise lag within each of these conditions was a randomly generated factor included to remove any regularity of stimulus presentation that might otherwise have become apparent to the subjects. For short lags, the mean lag between primes and targets and the mean list position of targets were 6 and 69 , respectively, in the neutral prime condition, 6 and 70 in the orthographic prime condition, and 6 and 66 in the prefixed BOSS condition. For long lags, the mean lag and target list position were 26 and 67 , respectively, in the neutral prime condition, 27 and 70 in the orthographic prime condition, and 25 and 69 in the prefixed BOSS condition.

Targets were randomly allocated to each lag condition in each stimulus group, giving a different selection of 12 targets in each lag condition in Group 1, Group 2, and Group 3. To enable equal representation of a target within each of the two lag conditions, the 12 primes (and their targets) in the short lag condition were alternated with the 12 primes (and their targets) in the long lag condition for consecutive subjects within a group. Thus, a target (e.g., monarch) would be presented in the short lag condition to half of the subjects in a group, and in the long lag condition to the other half. Over the three stimulus groups, each target thus contributed to performance in both lag conditions in the three prime conditions equally often.

\section{Apparatus}

The experiment was conducted under the control of an Acorn Atom microcomputer equipped with a real-time clock and a 9-in. high-resolution monochrome monitor (Hitachi VM-900). Each stimulus was fully plotted before screen presentation started, enabling each stimulus to be drawn on the screen at the speed of the scanning electron beam of the CRT. The timing of a response was initiated immediately after stimulus presentation.

\section{Procedure}

Thirty undergraduates from the University of Reading participated in the experiment, which lasted for half an hour and for which they were paid $\mathbf{1 0 . 5 0}$. All subjects were native speakers of English and had normal or corrected-to-normal vision. The subjects were randomly assigned to one of the three stimulus groups.

One subject produced a dramatically high error rate (36\%); this subject's data were discarded and replaced by those of a fresh subject.

The subjects were seated in a darkened room in front of a video monitor. Before each subject were two buttons, labeled "Yes" and "No," which the subjects operated with the index and middle fingers of their preferred hand.

On each trial, a small dot appeared in the center of the screen. When subjects were ready to receive a stimulus, they pressed either of the two response buttons. The screen then went blank for 500 msec during which time the stimulus was plotted. The screen was then enabled and the stimulus was presented in the center of the screen, centered around the position previously occupied by the dot. The stimulus remained on the screen for a maximum of $5 \mathrm{sec}$, after which time an error response was assigned to that trial. If a subject responded within this 5-sec period the type of response and the reaction time latency were stored by the computer and the display was terminated. After stimulus offset, the small dot reappeared in the center of the screen, signaling the availability of the next stimulus.

Before the experiment, the subjects were given the following instructions:

You will be shown a series of words and nonsense words presented in a random order. For each stimulus you must decide, as quickly as you can, whether that stimulus is a word or a nonsense word. If you think it is a word press the "Yes" button; if a nonsense word press the "No" button. If you take too long to decide, the stimulus will be removed from the screen. However, please try not to make too many errors.

The subjects were further informed that the experiment was designed to investigate speed and accuracy of response. This information was given both to enhance these instructions and to diminish the possibility of subjects' suspecting the experiment's real purpose, of which no mention was made.

The experiment was divided into two parts, separated by a 1 min break. In Part 1, subjects were shown, in a random intermixed order, 48 words and 48 nonwords from the practice list of 102 stimuli. This same list of stimuli was used in a practice session for all subjects.

The remaining six practice items were added to the beginning of the experimental session, Part 2, as a buffer, with no discrete event signaling a transition to the real experimental items. Although experimental items were presented in accord with the design of the 
experiment, each subject received a different random order of presentation.

Following the experimental trials, the subjects were encouraged to comment on the task. More specifically, they were asked to suggest possible purposes of the experiment that they may have perceived while taking part. Finally, they were told the real purpose of the experiment and were asked to divulge whether they had had any awareness of this purpose during the experiment.

\section{RESULTS}

The reaction time and error data for target words are shown in Table 1. Only data from targets both correctly categorized and preceded by a correctly categorized prime were included in the reaction time analysis, because for these items the probability that a response required the activation of the appropriate processes for lexical access should be higher than that for incorrectly categorized items, for which appropriate access may not have occurred.

All analyses of variance were computed with raw means of both subjects and items as units. Analyses included the factors of type of prime (neutral, orthographic, and prefixed BOSS) and length of lag (short or long).

For the reaction time data, no main effect of type of prime was found for subjects $\left[F_{1}(2,58)=2.22, p>.10\right.$, $M S e=11,498]$ or for stimuli $\left[F_{2}(2,46)=1.44, p>.10\right.$, $M S e=11,314]$. For length of lag, a main effect was found with subject means $\left[F_{1}(1,29)=6.73, p<.02, M S e=\right.$ $46,476]$, but not with item means $\left[F_{2}(1,23)=0.94\right.$, $p>.25, M S e=85,928]$. For the error data, no main effect or interaction was found (all $p s>.05$ ). However, the overall error rate of $5 \%$ may have been too low to support an analysis of variance. Consequently, a chi-square test of the number of subjects who made errors in each of the six conditions was also performed. Again, no significant differences were revealed (all $p s>.10$ ).

As suggested by the table, a type of prime $\times$ length of lag interaction was found for reaction times $\left[F_{1}(2,58)\right.$ $=4.91, p<.02, M S e=8,321 ; F_{2}(2,46)=4.60$, $p<.02, M S e=9,693]$.

Planned comparisons were performed between the subject means for each type of prime in both lag conditions. In the short lag condition, targets preceded by a prefixed BOSS prime were responded to more quickly than were those preceded by a neutral prime $\left[F_{1}(1,29)=6.85\right.$, $p<.02, M S e=20,525]$. Targets preceded by an orthographic prime were also responded to more quickly than were those preceded by a neutral prime $\left[F_{1}(1,29)\right.$ $=11.00, p<.005, M S \mathrm{e}=19,635]$. There was no sig-

Table 1

Mean Reaction Times (RT, in Milliseconds) and Percentage of Errors (E) for Targets at Each Lag in the Three Prime Conditions

\begin{tabular}{|c|c|c|c|c|c|c|}
\hline \multirow[b]{3}{*}{ Lag } & \multicolumn{6}{|c|}{ Type of Prime } \\
\hline & \multicolumn{2}{|c|}{ Neutral } & \multicolumn{2}{|c|}{ Orthographic } & \multicolumn{2}{|c|}{ Prefixed BOSS } \\
\hline & RT & $\mathrm{E}$ & $\mathrm{RT}$ & $\mathbf{E}$ & RT & $\mathrm{E}$ \\
\hline Short & 751 & 1 & 667 & 7 & 683 & 4 \\
\hline Long & 730 & 6 & 749 & 9 & 723 & 5 \\
\hline
\end{tabular}

nificant difference between the prefixed BOSS and orthographic prime conditions $(p>.25)$. In the long lag condition, no differences were found among the three prime conditions (for all comparisons, $p>.10$ ).

Within type of prime, the effect of length of lag was significant only for the orthographic prime condition $\left[F_{1}(1,29)=11.79, p<.005, M S \mathrm{e}=17,499\right]$. For all other types of prime, $p>.05$.

As a final check for any trade-off between errors and reaction time, all of the above analyses were repeated, using an analysis of covariance in which the influence of errors on reaction time was included as a covarying factor. The results reported above were replicated almost exactly, again showing that error rate had no systematic influence on reaction times.

\section{DISCUSSION}

Recall that this experiment was conducted to examine the appropriateness of the BOSS hypothesis for describing the process of lexical access. From the results obtained, it appears that when a word contains a sequence of letters that occurs in a later presentation of a different word, the repetition of this cluster across these two stimuli results in a facilitated response to the second word. Furthermore, the effect of this facilitation appears to diminish as the number of intervening trials increases, so that in the long lag condition (with a mean of 26 intervening trials) all three priming conditions elicited similar reaction times to targets. However, it is apparent that the facilitation observed was not restricted to the repetition of BOSS units, as the facilitation produced in the prefixed BOSS priming condition did not differ from that observed in the matched orthographic condition, in which primes and targets had no potential for accessing the same BOSS entry.

Before we examine this evidence more closely, the indication that the repetition effects observed were due to processes involved in the detection of orthographic units must be clarified, because orthographic units can also subserve phonological components, either pre- or postlexically (see Rosson, 1983), which may have contributed to the effects of facilitation that were found in this experiment. Therefore, an analysis was performed to assess the relationship between the phonological overlap between prime and target and the effect that this may have had upon target facilitation. Although they were matched for their orthographic content, the multiletter clusters present in both prefixed BOSS and orthographic primes typically varied in their phonological similarity to these same clusters in the target words (e.g., decant, vacant, canter). Consequently, if phonology was a potent influence on the facilitation effect, it seems reasonable to expect a systematic relationship between the degree of phonological similarity between a multiletter cluster in a prime and the same cluster in its target, and the effect of priming between these two words.

To determine whether such a relationship existed, 15 independent judges were asked to rate the shared mul- 
tiletter cluster in the prefixed BOSS and orthographic prime of each set for its phonological similarity to the same cluster in the target word for that set. Ratings were to vary between 0 and 5 , with 0 indicating no similarity and 5 indicating identical pronunciation. The judges were urged to review their ratings throughout the list to achieve an overall balance of ratings that best reflected their judgments. A mean rating of prime-target phonological overlap was then calculated for each prime and correlated with the mean reaction time for that target when it was preceded by that prime in the short lag condition of the lexical decision task. Because a higher value had been given to greater phonological overlap, a correlation suggesting a causal relationship between phonology and priming would have a negative value; a higher rating would indicate a higher priming potential, which should result in a lowered reaction time to a target if phonological priming was implicated. Using the Pearson product-moment coefficient, no significant relationship was found between the phonological overlap of primes and targets and reaction times for targets $[r(46)=+.16, p>.10]$. This result suggests that the effect of priming observed in the experiment was due to the orthography of both primes and targets, and not to their phonology.

It now seems reasonable to conclude that the priming effects observed in this experiment were the result of priming representations for orthographic sequences. Yet it must be emphasized that primes with the potential for priming within the constraints of the BOSS hypothesis showed no greater priming of target words than did their orthographically matched non-BOSS counterparts. If the BOSS hypothesis were correct, then repeated access to the same BOSS entry would be expected to produce additional facilitation beyond that observed for simple orthographic units. Consequently, the content of Taft's hypothesis for word recognition (Taft, 1979a, 1979b, 1981, 1984, 1985; Taft \& Forster, 1976) does appear to be open to question. Let us examine some possible reasons why this experiment does not support this view of the process of word recognition.

All primes in the prefixed BOSS condition required that this prefix be removed before the BOSS contained within a prime could be revealed. This was a necessary characteristic of stimuli designed to examine the major interacting aspects of the BOSS hypothesis. However, if this prefixstripping operation had not occurred, then it would be reasonable to suppose that no priming of an appropriate BOSS entry had taken place, and the similarity in performance between the prefixed BOSS and orthographic conditions would be at least partially explained. Henderson et al. (1984) and Taft (1985) reported evidence that prefixed nonwords are rejected more slowly than nonprefixed nonwords in a lexical decision task. In view of the apparent robustness of this effect, it would seem more certain that the present experiment provided a valid test of the BOSS hypothesis had this standard effect also been obtained. The nonword filler items used in this experiment were not designed to test for this effect, but 22 of the 24 prefixed nonwords (matched to the prefixed BOSS primes) were well matched to their nonprefixed nonword counterparts (which were matched to the orthographic primes) and thus provided a valid test of this effect. Prefixed nonwords took significantly longer to be rejected than nonprefixed nonwords (Wilcoxon; $z=1.74, p<.05$, one-tailed), producing mean response latencies of 1,022 and $959 \mathrm{msec}$, respectively. Prefixed nonwords also produced more errors $(4.2 \%$ vs. $1.7 \%$, Wilcoxon; $z=2.48, p<.01$, one-tailed). Consequently, the experiment replicated one prefix-stripping effect previously reported in the lexical decision literature (Henderson et al., 1984; Taft, 1985). However, the BOSS hypothesis predicts that a similar detection-of-prefixes effect should occur for words. To determine whether this effect did occur, subjects' performances with pseudoprefixed primes (e.g., demon) were compared to their performances with matched nonprefixed orthographic primes (e.g., lemon); the reasoning was that pseudoprefixed words should incur longer reaction times because of the inappropriate prefix-stripping process that is supposed to occur with these items (Taft, 1981). From the list of prefixed BOSS primes, seven items were selected as pseudoprefixed, and the reaction times to these items were compared with those to their matched orthographic controls. These items are the first seven pairs listed in the Appendix. Interestingly, there was no significant difference between the two lists of items (Wilcoxon; $z=1.36, p=.09$ ). Furthermore, the trend was for responses to pseudoprefixed items (mean RT = $631 \mathrm{msec}$ ) to be faster than responses to nonprefixed items (mean RT $=647 \mathrm{msec}$ ). Differences in the frequency of the words involved may have contributed to this result (the frequencies of the pseudoprefixed and orthographic primes were 89 and 40 occurrences per million, respectively; Carroll et al., 1971; Francis \& Kučera, 1982); however, Henderson et al. (1984) have also shown that pseudoprefixed words do not incur longer response latencies in the lexical decision paradigm. Clearly, results of this kind do not support the view contained within the BOSS hypothesis that the automatic removal of prefixes is a necessary preliminary stage for lexical access.

Despite the emphasis that the model places on the stripping of prefixes and the evidence just reported, it is not difficult to maintain the essential aspects of the BOSS hypothesis. A reformulation of the hypothesis that suggests that both intact and stripped versions of a stimulus are processed in parallel, rather than that the appropriate BOSS entry must be accessed on the second of two serial attempts, overcomes the need to show that pseudoprefixed items incur longer reaction times. However, even with this modification, it is conceivable that pseudoprefixed primes may be less appropriate than truly prefixed items for activating the internal representations for the BOSSs that were present in both the targets and primes of the present experiment. There are two possible reasons for this. First, if prefix stripping is a process that is not applied to all stimuli indiscriminately, then its employment should be apparent at least with truly prefixed items. Therefore, if the multiletter unit that is shared 
by a prefixed BOSS prime and its target is to be revealed at all, it may be that this appropriate parsing occurs only for truly prefixed primes. Second, even if pseudoprefixed and truly prefixed items are parsed, at some point, in much the same way, it may be that although the same BOSS entry is accessed in both cases, this access does not result in an excitatory trace for pseudoprefixed primes because the procedure is inappropriate for use with these items. Again, this suggests that priming will be observed only with truly prefixed primes and their targets.

If either or both of these possibilities are true, it seems reasonable to expect that the same BOSS entry would be employed for truly prefixed primes (e.g., decant) and their targets (e.g., canter). Judgments about the nature of a prefix can vary considerably, but 12 of the primes in the prefixed BOSS condition appear to constitute a good representative sample of truly prefixed words. These primes are the final 12 listed in the Appendix. If these primes are well suited to the analysis proposed by the BOSS hypothesis (and it is difficult to see how they might not be), then these items, at least, should have facilitated the recognition of their targets to a greater degree than did their matched orthographic controls. A comparison of the facilitation produced by these two types of prime showed no significant difference, for either short or long lags (Wilcoxon; both $z \mathrm{~s}<0.62, p\rangle .25$, one-tailed). For the short lag condition, the mean target response time produced by the prefixed BOSS primes $(699 \mathrm{msec}$ ) actually exceeded that produced by their orthographic controls by (a nonsignificant) $35 \mathrm{msec}$. This result is difficult to encompass within the model of lexical access provided by the BOSS hypothesis, for even when an analysis is restricted to stimuli that are most appropriate to the type of processing that the model proposes, no evidence is obtained for its support.

The lexical decision paradigm was used in the present experiment to provide a reliable comparison between the evidence obtained here and that on which the BOSS hypothesis is based, which has been derived almost exclusively from lexical decision experiments. It may be that, for some reason, the internal representations for BOSSs do not enjoy facilitation or that the present experiment was not sufficiently sensitive to tease out priming effects that were specific to these units. But it is difficult to see why this should be, since the paradigm seems well suited to exposing the effects of orthographic repetition. Why should the influence of purportedly critical units in word recognition not be observed, when influences of simple orthographic repetition were so apparent? At this point it must be acknowledged that indications from other sources also show that the BOSS hypothesis is less than robustly supported. In particular, Lima and Pollatsek (1983) found serious inconsistencies in the proposal that lexical access is mediated by an access code that conforms to the BOSS of a word; their findings suggest that perhaps the failure of the present experiment to find a priming effect specific to BOSSs is due to the lack of actual lexical representations for these syllables (see also Smith, Meredith, Pattison, \& Sterling, 1984). As the present experiment shows no evidence to suggest that any of the components of the BOSS hypothesis are used for perceiving words, this conclusion is certainly not without merit.

However, although the present study found no evidence in support of the BOSS hypothesis, it was apparent that the repetition of multiletter units across two stimuli did facilitate the response to the second item. Furthermore, these effects do not appear to be under the strategic control of subjects, as no subject reported discerning the repetition aspect of the experiment. In addition, most subjects were doubtful that repeated multiletter units should aid recognition, which suggests that their reported unawareness of this experimental manipulation was accurate. Interestingly, Henderson et al. (1984) found that orthographic priming in a lexical decision task actually increased lexical decision times to targets, relative to a neutral control condition. However, in their experiment, stimuli were presented in distinct pairs, with a target directly following its prime. Furthermore, in addition to orthographic and neutral priming conditions, other word pairs were either semantically or morphemically related. Consequently, even if their subjects were not explicitly informed of the repetition aspect of the experiment, this repetition may have become apparent after only a few trials and subjects may have then attempted to predict targets from the nature of the prime. This view gains some support from the finding that in all four conditions, targets presented $4 \mathrm{sec}$ after their primes were responded to, on average, $50 \mathrm{msec}$ faster than were those presented at a prime-target interval of just $1 \mathrm{sec}$, suggesting that when more time was available to predict a set of potential targets, target reaction time decreased. A strategy of this kind may have removed the effect of orthographic priming that was observed in the present study.

It is perhaps interesting to consider how the positioninsensitive facilitation of multiletter clusters that has been observed in the present experiment can be encompassed within models of word recognition in which individual position-specific channels of letter detection enable access to an appropriate lexical entry (e.g., Johnston \& McClelland, 1980; McClelland \& Rumelhart, 1981; Rumelhart \& McClelland, 1982). In models of this type, each serial position within a word is first processed by a position detector. Each letter is then detected through an array of letter detectors that is specific to that serial position. Thus, any facilitation obtained between two words such as have and cave may be explained by the repeated employment of three letter detectors, as the same three letters occur in the same three positions in both words. However, it is clear from an inspection of the stimuli used in the present experiment that letters shared between primes and target do not share the same serial position (e.g., demon/lemon, monarch). Consequently, the repeated employment of a selection of position-specific letter detectors seems an unlikely explanation of the priming effects that were observed. However, words may not be perceived through the activation of individual letter detectors specific to each serial position. Rather, word recognition may require that larger features, positionally 
unrestricted and encompassing several letters, be perceived, and the same psychological representation for any such feature may be used whenever it is encountered, irrespective of its position within a word. Thus, the perception of lemon and monarch would involve the repeated use of the same representation for the letter sequence common to both items (mon), resulting in the priming effect that was observed.

However, it may be dangerous to conclude that the evidence from the present experiment necessarily indicates the process of word recognition. A recent analysis of the lexical decision paradigm suggests that the effect of the familiarity of a letter string may be attributed to the decision stage of a response, rather than to processes involved in actually accessing the appropriate lexical entry (Balota \& Chumbley,1984). Consequently, it is uncertain that the orthographic priming effects observed in the present study reflect aspects of the recognition of words. Yet these effects may provide an indication of processes that are critically involved in making lexical decisions.

In view of the precautions taken in the stimulus selection procedure, it is difficult to see how the repetition aspect of this experiment might have induced processes not normally used in deciding the lexical nature of a stimulus. Indeed, the stimulus set of a lexical decision task not concerned with the study of repetition effects may nevertheless contain a number of multiletter units that are repeated across items. This may often be regarded as a natural reflection of the repeated exposure to such units in our everyday linguistic environment, and may be a characteristic that increases the similarity between the experiment and natural reading. However, unlike natural reading, the lexical decision task generally requires the subject to perform a precise letter-by-letter analysis of a stimulus in order to decide whether or not it is a word. This kind of specific analysis may be completely unnecessary in a reading environment, in which nonwords are rarely encountered and lexical decisions do not have to be made. Irrespective of whether multiletter units are repeated in an experiment, the identification of many sequences of letters may be extraordinarily enhanced by the requirement to make lexical decisions, and it is perhaps only when these units are the focus of experimental attention (as in the present study) that this enhancement becomes apparent. Consequently, although the repetition effects observed here may be the result of facilitating the perception of multiletter units by priming lexical representations for these features, it is also plausible that these results reflect the lexical decision task's overemphasis on the subjective analysis of a stimulus into a sequence (or sequences) of letters.

This view gains support from a study by Oliphant (1983), who found a priming effect between two presentations of a word only when both presentations occurred within a lexical decision task. When the first presentation occurred in a more natural setting (within a piece of text), no facilitation was observed when subjects responded to the same item when it was subsequently presented for lexical decision. The argument presented here suggests that the degree of stimulus analysis required to perform the lexical decision task may involve an unusually thorough analysis of the individual letter content of each stimulus. Thus, words and nonwords may be distinguished more accurately. Yet, in the study by Oliphant, the extent of this analysis may have emphasized the identity of each stimulus and produced the effect of repetition that was observed when both presentations occurred in the lexical decision experiment. However, it may be that when the first presentation of a word did not require a lexical decision, the level of stimulus analysis was far less extensive, although sufficient for lexical access to occur, and did not facilitate a later lexical decision to the same item.

This view of the way in which lexical decisions are accomplished adds its own caveat to theories of word recognition developed predominantly from evidence provided by the lexical decision paradigm. If the perception of stimuli in lexical decision experiments requires an unnatural emphasis on the letter-by-letter construction of items, then the influence of certain letter sequences is a function not of normal lexical processes but of the demands of the task employed. Therefore, the conclusion that certain letter sequences are invariably used in the recognition of words may be false. Although it could be suggested that certain letter sequences (e.g., BOSSs) are still important under these conditions, such an observation may offer misleading indications about the way in which words are perceived in more natural reading situations.

\section{REFERENCES}

Balota, D. A., \& Chumbley, J. I. (1984). Are lexical decisions a good measure of lexical access? The role of word frequency in the neglected decision stage. Journal of Experimental Psychology: Human Perception \& Performance, 10, 340-357.

Carroll, J. B., Davies, P., \& Richman, B. (1971). The American heritage word frequency book. Boston: Houghton Mifflin.

FrancIS, W. N., \& KUČERA, H. (1982). Frequency analysis of English usage: Lexicon and grammar. Boston: Houghton Mifflin.

Gibson, E. J., \& Guinet, L. (1971). Perception of inflections in brief visual presentations of words. Joumal of Verbal Learning \& Verbal Behavior, 10, 183-189.

HANSEN, D., \& RoDGERS, T. S. (1968). An exploration of psycholinguistic units in initial reading. In K. S. Goodman (Ed.), The psycholinguistic nature of the reading process (pp. 59-102). Detroit: Wayne State University Press.

Henderson, L., Wallis, J., \& KNIGHT, D. (1984). Morphemic structure and lexical access. In H. Bouma \& D. G. Bouwhuis (Eds.), Attention and performance $X$ : Control of language processes (pp. 211 226). London: Erlbaum.

Johnston, J. C., \& MCCLELland, J. L. (1980). Experimental tests of a hierarchical model of word identification. Joumal of Verbal Leaming \& Verbal Behavior, 19, 503-524

Lima, S. D., \& Pollatsek, A. (1983). Lexical access via an orthographic code? The Basic Orthographic Syllabic Structure (BOSS) reconsidered. Journal of Verbal Learning \& Verbal Behavior, 22, 310-332.

MCClei.land, J. L. \& \& Rumelhart, D. E. (1981). An interactive activation model of context effects in letter perception: Part 1. An account of basic findings. Psychological Review, 88, 375-407.

Meyer, D. E.. Schvanevel.dT. R. W. \& Ruddy, M. G. (1974). Function of graphemic and phonemic codes in visual word recognition. Memory \& Cognition, 2. 309-321 
Meyer, D. E., Schyaneveldt, R. W., \& Ruddy, M. G. (1975). Loci of contextual effects on word recognition. In P. M. A. Rabbitt \& S. Dornic (Eds.), Attention and performance V (pp. 98-118). New York: Academic Press.

MurRell, G. A., \& Morton, J. (1974). Word recognition and morphemic structure. Joumal of Experimental Psychology, 102, 963-968.

NeELY, J. H. (1977). Semantic priming and retrieval from lexical memory: Roles of inhibitionless spreading activation and limited capacity attention. Joumal of Experimental Psychology: General, 106 , 226-254.

OLiphant, G. W. (1983). Repetition and recency effects in word recognition. Australian Journal of Psychology, 35, 393-403.

Rosson, M. B. (1983). From SOFA to LOUCH: Lexical contributions to pseudoword pronunciation. Memory \& Cognition, 11, 152-160.

Rubenstein, H., Lewis, S. S., \& Rubenstein, M. A. (1971). Evidence for phonemic recoding in visual word recognition. Journal of Verbal Learning \& Verbal Behavior, 10, 645-657.

Rubenstein, H., Richter, M. L., \& Kay, E. J. (1975). Pronounceability and the visual recognition of nonsense words. Journal of Verbal Learning \& Verbal Behavior, 14, 651-657.

Rubin, G. S., Becker, C. A., \& Freeman, R. H. (1979). Morphological structure and its effect on visual word recognition. Journal of Verbal Learning \& Verbal Behavior, 18, 757-767.

Rumelhart, D. E., \& MCClellaNd, J. L. (1982). An interactive activation model of context effects in letter perception: Part 2. The contextual enhancement effect and some tests and extensions of the model. Psychological Review, 89, 60-94.

Shulman, H. G., Hornak, R., \& Sanders, E. (1978). The effects of graphemic, phonetic, and semantic relationships on access to lexical structures. Memory \& Cognition, 6, 115-123.

Smith, P. T., Meredith, T. R., Pattison, H. M., \& Sterling, C. M. (1984). The representation of internal word structure in English. In
L. Henderson (Ed.), Orthographies and reading (pp. 103-120). London: Erlbaum.

SPOEHR, K., \& SMITH, E. (1973). The role of syllables in perceptual processing. Cognitive Psychology, 5, 71-89.

Stanners, R. F., Neiser, J. J., Hernon, W. P., \& Hall, R. (1979). Memory representation for morphologically related words. Journal of Verbal Learning \& Verbal Behavior, 18, 399-412.

Stanners, R. F., Neiser, J. J., \& Painton, S. (1979). Memory representation for prefixed words. Joumal of Verbal Learning \& Verbal Behavior, 18, 733-743.

TAFT, M. (1979a). Lexical access via an orthographic code: The Basic Orthographic Syllabic Structure (BOSS). Journal of Verbal Learning \& Verbal Behavior, 18, 21-39.

TAFT, M. (1979b). Recognition of affixed words and the word frequency effect. Memory \& Cognition, 7, 263-272.

TAFT, M. (1981). Prefix stripping revisited. Journal of Verbal Learning \& Verbal Behavior, 20, 289-297.

TAFT, M. (1984). Exploring the mental lexicon. Australian Journal of Psychology, 36, 35-46.

TAFT, M. (1985). The decoding of words in lexical access: A review of the morphographic approach. In D. Besner, T. G. Waller, \& G. E. MacKinnon (Eds.), Reading research (Vol. 5, pp. 83-123). Orlando, FL: Academic Press.

TAFT, M., \& FoRSTER, K. I. (1975). Lexical storage and retrieval of prefixed words. Journal of Verbal Learning \& Verbal Behavior, 14, 638-647.

TAFT, M., \& Forster, K. I. (1976). Lexical storage and retrieval of polymorphemic and polysyllabic words. Journal of Verbal Learning \& Verbal Behavior, 15, 607-620.

VAN DER MOLEN, H., \& MORTON, J. (1979). Remembering plurals: Unit of coding and form of coding during serial recall. Cognition, 7 , $35-47$.

\section{APPENDIX}

Word Sets Used in the Experiment

\begin{tabular}{clllllll}
\hline $\begin{array}{c}\text { Neutral } \\
\text { Prime }\end{array}$ & $\begin{array}{c}\text { Orthographic } \\
\text { Prime }\end{array}$ & $\begin{array}{c}\text { Prefixed BOSS } \\
\text { Prime }\end{array}$ & Target & \multicolumn{3}{c}{$\begin{array}{c}\text { Reaction Time } \\
\text { (in } \text { Milliseconds) }\end{array}$} \\
\hline pedal & lemon & demon & monarch & 597 & 670 & 637 \\
salute & novice & device & vicar & 735 & 758 & 665 \\
focus & legal & regal & gala & 727 & 653 & 583 \\
rapid & civil & devil & vile & 842 & 669 & 709 \\
secret & parson & person & sonic & 788 & 709 & 566 \\
topic & level & revel & velocity & 761 & 589 & 808 \\
mural & pivot & divot & vote & 695 & 519 & 736 \\
rampart & solvent & convent & venture & 751 & 641 & 601 \\
secure & severe & revere & verify & 882 & 646 & 754 \\
parcel & hermit & permit & mitre & 783 & 820 & 870 \\
cavern & silent & relent & lentil & 753 & 692 & 812 \\
vocal & habit & debit & bitch & 565 & 629 & 684 \\
hazard & cutest & detest & testify & 1113 & 731 & 806 \\
robust & vacant & decant & canter & 789 & 737 & 618 \\
cabbage & suspect & respect & spectate & 1045 & 873 & 717 \\
garage & palate & relate & latch & 675 & 507 & 607 \\
bonus & lapel & repel & pelican & 986 & 658 & 989 \\
menace & satire & retire & tirade & 767 & 711 & 585 \\
forest & saturn & return & turnip & 539 & 660 & 548 \\
linen & wafer & defer & ferocity & 736 & 770 & 711 \\
octave & umpire & expire & pirate & 583 & 646 & 784 \\
alters & rodent & indent & dental & 605 & 618 & 502 \\
captions & viscount & discount & country & 539 & 660 & 548 \\
mustard & culvert & convert & vertical & 623 & 594 & 816 \\
\hline
\end{tabular}

Note-Figures after each target show mean reaction times to that target at short lags in the neutral, orthographic, and prefixed BOSS conditions, respectively. 